\title{
Diagnosing of some hepatic lesions from light microscope images based on morphological and texture features
}

\author{
Zamen F. Jabr, Mohammed A.A. Hasan \\ College of education for women, University of Thi-qar (UTQ), Iraq
}

\begin{tabular}{l}
\hline \hline Article Info \\
\hline Article history: \\
Received Sep 2, 2019 \\
Revised Nov 3, 2019 \\
Accepted Nov 17, 2019 \\
\hline
\end{tabular}

\section{Keywords:}

GLCM

Hepatic lesions diagnosing

Light microscope image

Morphological cells features

Neural network

\begin{abstract}
One of the common problems observed in medicines is hepatotoxicity as liver play mainly role in metabolizes the herbal medicines. Although, the acceptance of herbal medicines is growing nowadays still there is an absence of knowledge about their toxicological properties and the right use being a hepatotoxic. This paper presents method to detect and diagnoses liver lesions in four types: necrotic cells, fatty degenerative cells, hepatocellular hypertrophic cells and congested cells using image processing techniques. The method is proposed to perform two tasks the first is conclude whether the liver image is normal or abnormal the second if abnormal state is detected then diagnosis lesions type must performs. The method progresses in many steps are preprocessing, features extraction, classification and lesion diagnosing. Grey level co-occurrence Matrix (GLCM) technique is utilize to concentrate features to distinguish between normal and abnormal case using neural network classifier if abnormal state is detected the method feedback with colour image to analyse cells shape and image intensity colour to determine which type of diseases founded in image based on statistical and morphological features of cells. The method tested on 107 images it is got on the accuracy $100 \%$ in classification and $95 \%$ in diagnosing.
\end{abstract}

Copyright (C) 2020 Institute of Advanced Engineering and Science. All rights reserved.

\section{Corresponding Author:}

Zamen Fadhel Jabr, College of education for women, University of Thi-qar, Iraq. Email: Zamen-fadhel@utq.edu.iq

\section{INTRODUCTION}

Now days there is increasing in popularity of herbal medicines but still there is a shortage of information about their toxicological side view and suitable use being a hepatotoxic; repeatedly usage of these medicines imply many risk factors involve " diabetes mellitus, renal disease, liver diseases, rheumatic diseases, heart diseases, genetic factors like age issues and gender pregnancy, AIDS, alcohol abuse, psoriasis lack of food and adiposity" [1, 2].

The development of hepatotoxicity of metabolic-toxic for resulted from many reasons such as "shrank secretion of lipoproteins, inhibition of biliary secretion, inhibition of fatty acid mitochondrial $\beta$-oxidation lesions or Ito cells activation, metabolite-mediated toxicity and endothelial" [3]. In addition, Kupffer cells initiation result in the discharge of pro-inflammatory mediators (cytokines and chemokines) and cytotoxic mediators (reactive oxygen species), respectively [4]. However, the comprehensive mechanism of hepatotoxicity of many drugs containing herbal is still stay unidentified and reactive metabolite's formation is rather frequent $[3,5]$.

There are numerous methods that have been suggested by researchers deal with CT liver images these methods are applied to detect hepatic lesions proportion to the tissue natures (Normal, Abnormal) based on important features are took out from the lesion area. Our proposed method utilizes light microscope image to detect hepatic lesion and determines counts and poisons of abnormal cells. 
A. Sayed etal [6] proposed an automatic system to liver diseases diagnosis based on ultrasound images firstly the researchers interest in outlining the counter of the Region of Interestd (ROI) then the extracted features quantified using texture analyses which are computed based on statistics measures applied on gray scale image the classification of region is normal or not is done based on Neural network the classification accuracy is $96.125 \%$.

A. H. Ali and E. M. Hadi [7] proposed segmentation method to extract liver tumour from CT image the basic principle of the suggested algorithm is integrating the contour of the tumour with K-means clustering. Geometrical parameters of the tumour is computed to define the type of tumour was malignant, benign or Cyst.

Y. A. Deore and N. D.Ghuse [8] proposed method to detecting and diagnosing liver tumour region with lesion from Computed Tomography (CT) images their method utilize K-means clustering algorithm and Haar wavelet transform to compute the range values which determine whether input image is having cancer or not the precision of this method did not mention in their paper.

Pruthvi P R et al [9] based work CT image to detect the liver cancer tumor using support vector machine classifier to isolate the liver region from other overlapped organs and tissues in CT image based on texture information to the tumour tissues.

H. Alahmer and A. Ahmed [10] proposed system to extracting the main features took from multiple ROIs in order to diagnose the type of liver abrasion to Benign or Malignant. The researchers divided the lesion region into three parts inside part, outside part, and the border part. The features extracted from three parts which is consider novelty in their work. SVM used as classifier tool to determine if the lesion is benign or malignant accuracy of this system over $98 \%$.

T. Prakash [11] use CT abdominal image to identifying liver an adaptive threshold is used to isolate liver pixels from CT image in order to decrease computation time the cropping process is applied on image the edges of liver organ on CT image is detected using Soble algorithm. Fuzzy C Mean clustering (FCM) technique is used to tumour extraction from the liver. The accuracy of this method is $90 \%$.

A. Das et al [12] proposed system named as watershed Gaussian based deep learning (WGDL) to detect the cancer in CT images when tumor segmentation is performed texture features is extracted then these features fed to deep neural network to determine which type of cancer founded in CT image. The classification accuracy of this method is $99.38 \%$.

The purpose of current paper is to prepare automatic classification and diagnosing system to some type of hepatic lesion diseases based on light microscope image which is give digital information about the shape and color of liver cells in normal abnormal case.

The remaining of this paper is ordered as following, Section 2 introduce dataset description, Section 3 is the steps of the proposed method, Section 4 resultsd and discussiond, and finally Section 5 is the conclusions.

\section{DATASET DESCRIPTION}

The dataset is used in this paper consists of 107 Olympus light microscope images to normal and abnormal liver tissue cell. There is 7 image have normal liver tissue while the remaining 100 image have abnormal liver tissue. All abnormal images have four classe of lesions Congestion regions, Fatty degeneration (FD), hepatocellular necrotic (HN), hepatocellular hypertrophic (HHP) cells. The medical definition of these lesions as follow:

a) Congestion: Can be describe as an excessive or abnormal gathering of blood or other fluid in body fragment or blood vessel [13].

b) Fatty Degeneration: Deterioration of the cells of the body, accompanied by the formation of fat globules within the diseased cells [2].

c) Hepatocellular Necrosis: Death of a circumscribed portion of animal or plant tissue [14].

d) Hepatocellular Hypertrophy: The term can have various connotations including an increase in the weight of the organ (liver hypertrophy), an increase in the average size of the hepatocytes [15].

\section{THE PROPOSED METHOD}

In this paper the diagnosing method of hepatic lesions passes in four major steps which are preprocessing, features extraction, classification and diagnosing step. Figure 1 shows flow chart of the proposed method. More details about these steps show in the following sub items. 


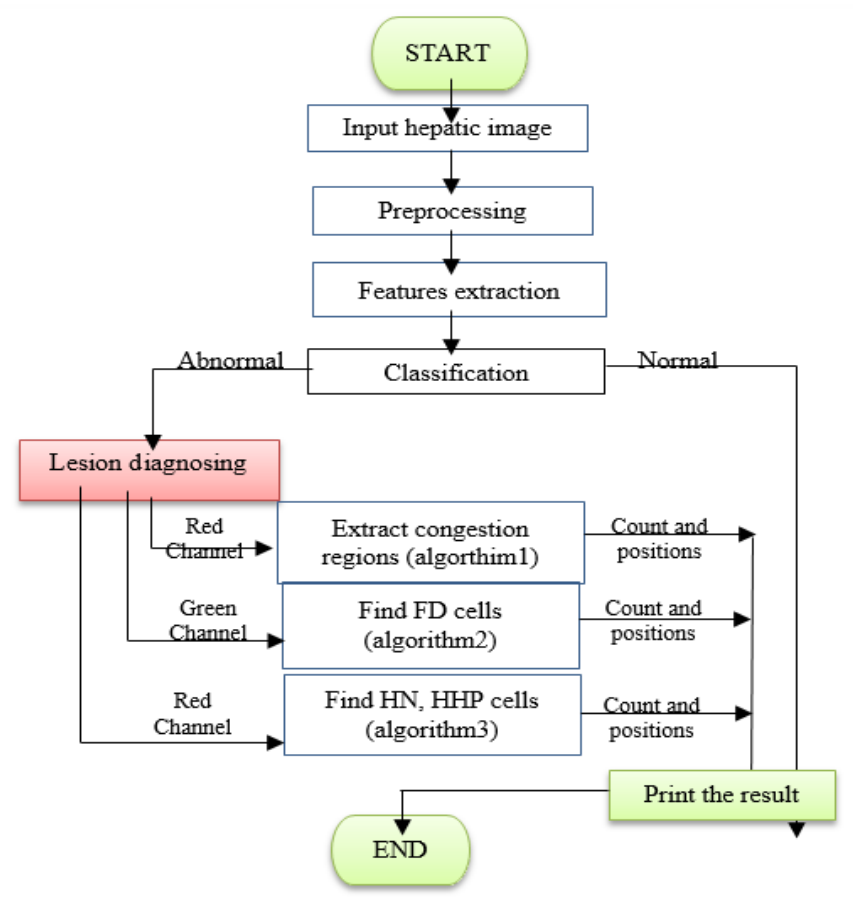

Figure 1. Flow chart of the proposed method

\subsection{Input hepatic image}

First stage in any recognition system is read images also our method starts in reading of liver cells image with two kinds normal or abnormal. Abnormal liver images involve four types of lesions: Congestion regions, FD cells, HN cells, HHP all image in tif format. Figure $2 \mathrm{~A}, \mathrm{~B}$ shows normal and abnormal liver tissue images receptively.

\section{B}
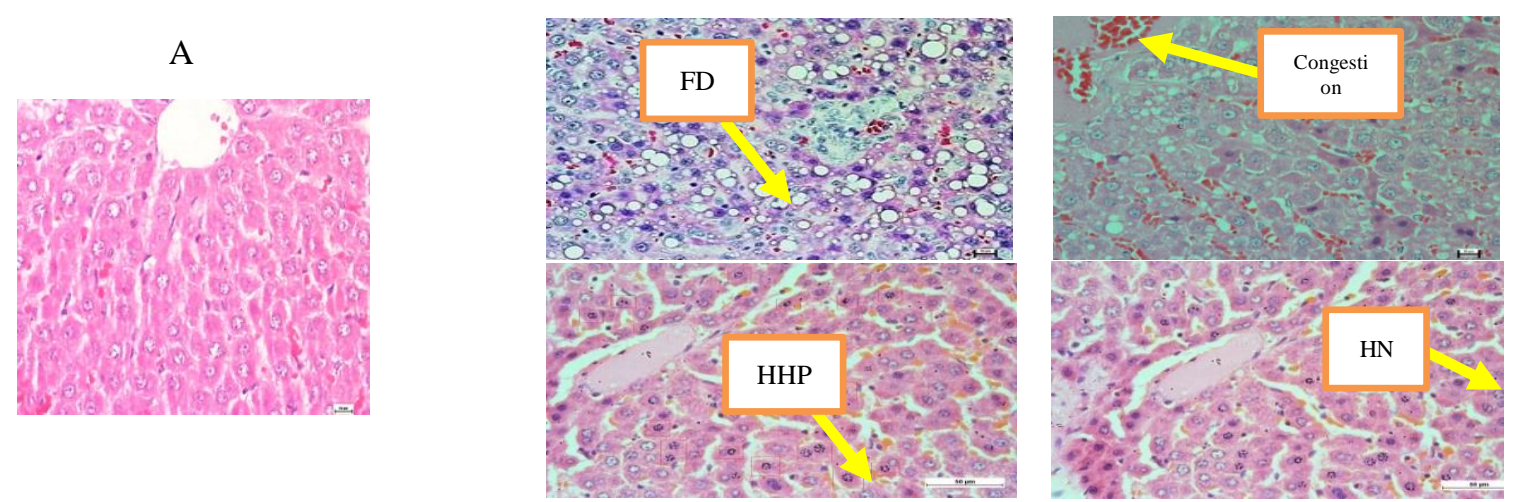

Figure 2. Liver tissues (A) normal tissue (B) abnormal tissue

\subsection{Preprocessing}

The color image converted to gray level by computing the average of pixels in three layers (red, green and blue) in color image; so image is converted from 3dimention image to 2D image. After that median filter with size window $3 \times 3$ applied to gray image in order to remove noise resulted from image capturing process. Median filter is benefit tool to remove salt and pepper noises appear during image capturing.

\subsection{Features Extraction}

Grey Level Co-occurrence Matrix (GLCM) method proposed by Haralick can be defined as statistical method used to compute number of texture features for an image. GLCM is also has another name is gray level Dependency Matrix. It is defined as "A two dimensional histogram of gray levels for a pair of pixels, which 
are separated by a fixed spatial relationship". To compute the GLCM features of an image the displacement vector $\mathrm{d}$ must be used which is defined by its radius $\delta$ and orientation $\theta[16,17]$. Haralick mined thirteen texture features from GLCM for an image in this paper only four features from GLCM used which are contrast, correlation, energy and homogeneityd which are defined as follow:

a) Contrast: is a measure of intensity or grey-level variations between the reference pixel and its neighbour $[18,19]$.

b) Correlation: this feature detects how a reference pixel is associated (correlated) to its neighbour where value 0 is uncorrelated pixels, 1 is perfectly correlated pixels [20].

c) Energy: the uniformity of colours in an image can be measured using energy. When pixels are very similar in it is values, the Angular Second Moment (ASM) which is also called uniformity or energy, value will be large. [21, 22].

d) Homogeneity: this feature gets the measures of the closenessd of the distribution of the GLCM elements to the GLCM diagonal [20].

GLCM computation based on major factor which is number of grey levels; the maximum gray value of the pixel used to determine the dimension of a GLCM [22]. The method which is proposed in this paper based on GLCM algorithm to extract texture features vector of hepatic image. This vector implies 16 features values which represent the GLCM features Homogenous, Energy, Contrast, and Correlation in four orientation degrees $\theta(0,45,90$, and 135) for each one of these features. Based on this features vector the classifier in the next step decides whether the hepatic image is normal or abnormal. The following Table 1 shows features vector to normal and abnormal liver images.

Table 1. GLCM Features Vector

\begin{tabular}{ccc}
\hline Features vector & Normal & Abnormal \\
\hline Contrast $(\theta=0)$ & 182.6398682 & 11.18543024 \\
Correlation $(\theta=0)$ & 0.850780605 & 0.986929576 \\
Energy $(\theta=0)$ & 0.000584466 & 0.003072883 \\
Homogeneity $(\theta=0)$ & 0.306766828 & 0.533732319 \\
Contrast $(\theta=45)$ & 276.0469396 & 19.74505487 \\
Correlation $(\theta=45)$ & 0.774137178 & 0.976930986 \\
Energy $(\theta=45)$ & 0.000419682 & 0.002255056 \\
Homogeneity $(\theta=45)$ & 0.25110047 & 0.465037731 \\
Contrast $(\theta=90)$ & 144.6544775 & 10.49917926 \\
Correlation $(\theta=90)$ & 0.8816971 & 0.987732965 \\
Energy $(\theta=90)$ & 0.0006398 & 0.003132413 \\
Homogeneity $(\theta=90)$ & 0.321465076 & 0.53909919 \\
Contrast $(\theta=135)$ & 278.3287798 & 19.93487223 \\
Correlation $(\theta=135)$ & 0.772271309 & 0.97670921 \\
Energy $(\theta=135)$ & 0.000420828 & 0.002263376 \\
Homogeneity $(\theta=135)$ & 0.250715116 & 0.46535598 \\
\hline
\end{tabular}

\subsection{Classification}

In order to classify the hepatic image normal or abnormal case an artificial neural network (ANN) used as classifier tool in our method. ANN can be described as a one type of artificial intelligence techniques which attempt to simulate the activities of nervous system in the human brain. The architecture of ANN consists of a number of computational units which is called neurons; these neurons are arranged in multiple layers. These layers are named input layer, hidden layer, and output layer. The weights of Network that are used to connect each neuron to other neuron in the next layers these weights are adjustable through a learning process until riches to desired output. In order to obtain the output of ANNs the hidden and output layers multiply each input by its network weights and then do summation on these multiplying which then the result passed via a transfer (activation ) function to get the output value [23, 24].

There are many type of learning algorithm used to training the ANN in this method Resilient backpropagation (Rprop) algorithm used to training ANN to be used in classification stage. Rprop is a learning algorithm which is the best algorithm in terms of combining correctness, speed as well as strength with respect to the training parameters. The Rprop is a local adaptived learning algorithm; the notion of this algorithm simple is to minimize the harmful influence that present due to the size of the partial derivative on the weight step [25].The architecture shape of ANN which is used in our method was 16 neurons in input layer this number match number of extracted features from GLCM algorithm and one hidden layer has 20 neurons and 2 neurons in output layer one for normal liver state and the second to abnormal state. Figure 3 shows the construction of ANN which is used in classification stage. 
The target matrix which is used to train this network has number of Rows equivalent to a number of information vectors of liver image features vector and columns equivalent to the class number equivalent to that input vector. In this matrix each rows contains on zeros value with a 1 value in an element with position $i$, where $\mathrm{i}$ is the class numberd that we need to treat it. The dataset of liver tissue images divided into two groups one for training with a rate $30 \%$ of overall dataset and second group for testing with a rate $70 \%$. The highest correct classification rate in this method was $100 \%$ in the training and testing groups. Table 2 shows the confusion matrix resulted in the testing group.

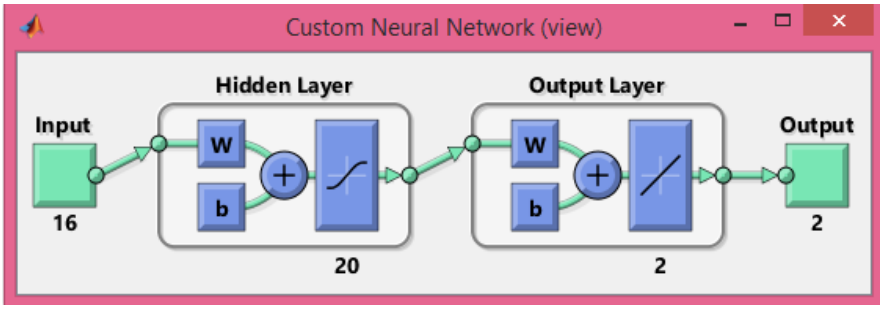

Figure 3. Architecture of ANN
Table 2. Confusion Matrix for Testing Group

\begin{tabular}{cccc}
\hline Output Class 1 & 5 & 0 & $100 \%$ \\
& $6.8 \%$ & $0.0 \%$ & $0.0 \%$ \\
Output Class 2 & 0 & 69 & $100 \%$ \\
& $0.0 \%$ & $93.2 \%$ & $0.0 \%$ \\
Overall & $100 \%$ & $100 \%$ & $100 \%$ \\
Percentage & $0.0 \%$ & $0.0 \%$ & $0.0 \%$ \\
& Target & Target & \\
& Class 1 & Class 2 & \\
\hline
\end{tabular}

\subsection{Lesions Diagnosing}

If abnormal case founded in classification stage we return to feedback the system with color image and analysis it's color layers to determine which type of lesion founded in it. The three channels of color image is isolated each one in separate gray image in order to find the positions and number of each type of lesions. Red and green channels are used to find major features which distinguish type of lesion. Algorithm 1 is proposed to extract the congestions region from hepatic image.

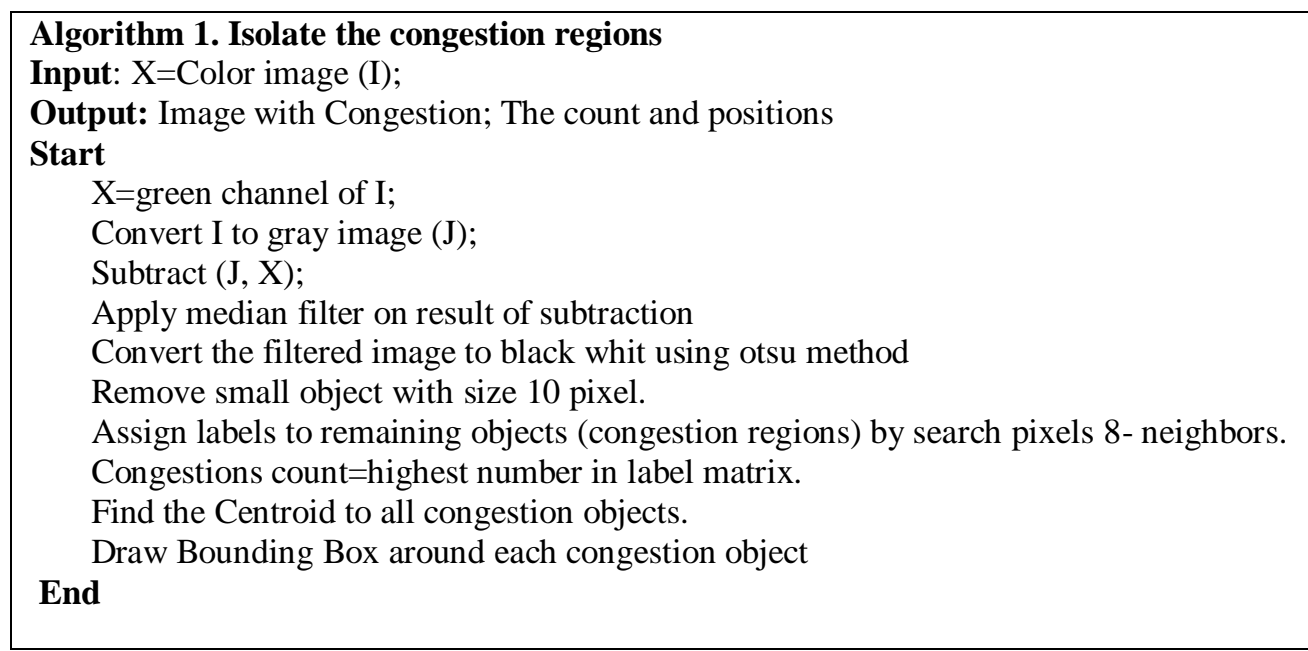

To find the hepatocellular hyper trophy cells (HHP) and necrotic cells (HN) in abnormal case the red channel is isolated from image then search is done about HN cell start by look to the intensity color of each region then nucleus number of each liver cells is computed to determine if the case of lesion is (HHP) or (HN) if the number of nucleus is more than one the state diagnosis is (HHP) else the state diagnosis is (HN). Euler number is used to computing number of gaps found in each cell where these resulted gaps match number of nucleus in original liver tissue image; if this number more than one then the cell has HHP lesion. An algorithm 2 outlines the steps to find (HHP) and (HN) cells. 


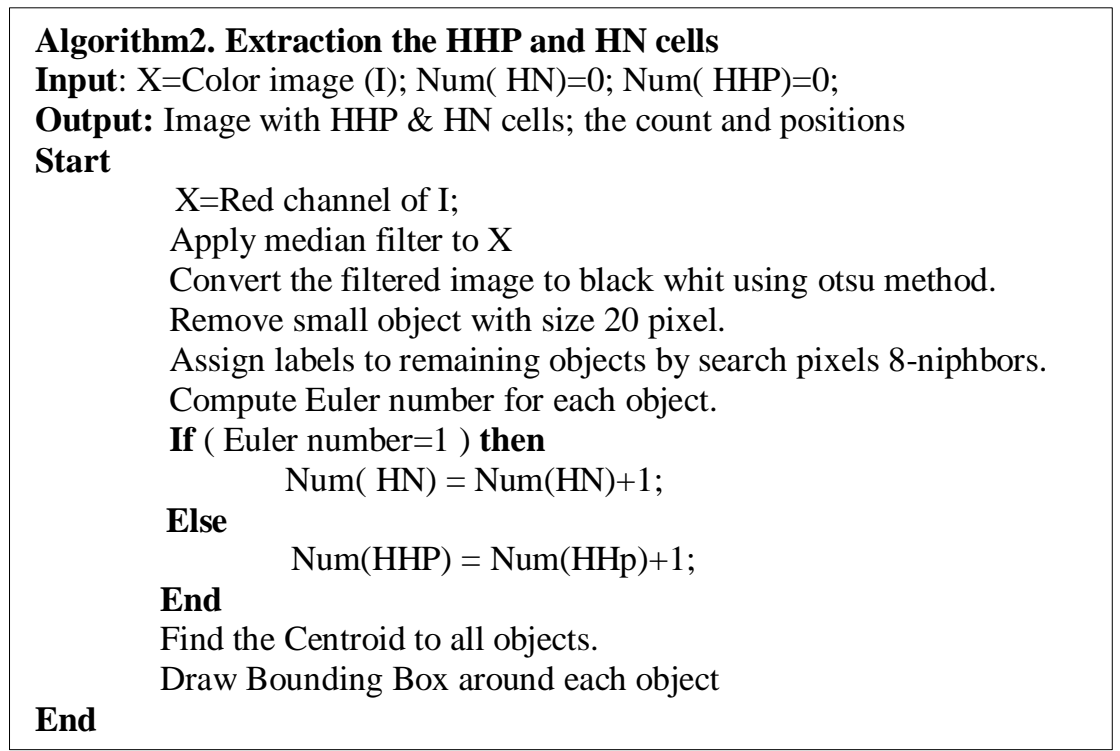

Third case fatty degeneration cell founded in green channel obviously so algorithm 3 developed to extract these fatty cells this algorithm use the morphological operation closing with disk shape to detect fatty cell also it is search about circular object shape with Eccentricity less than 0.6.

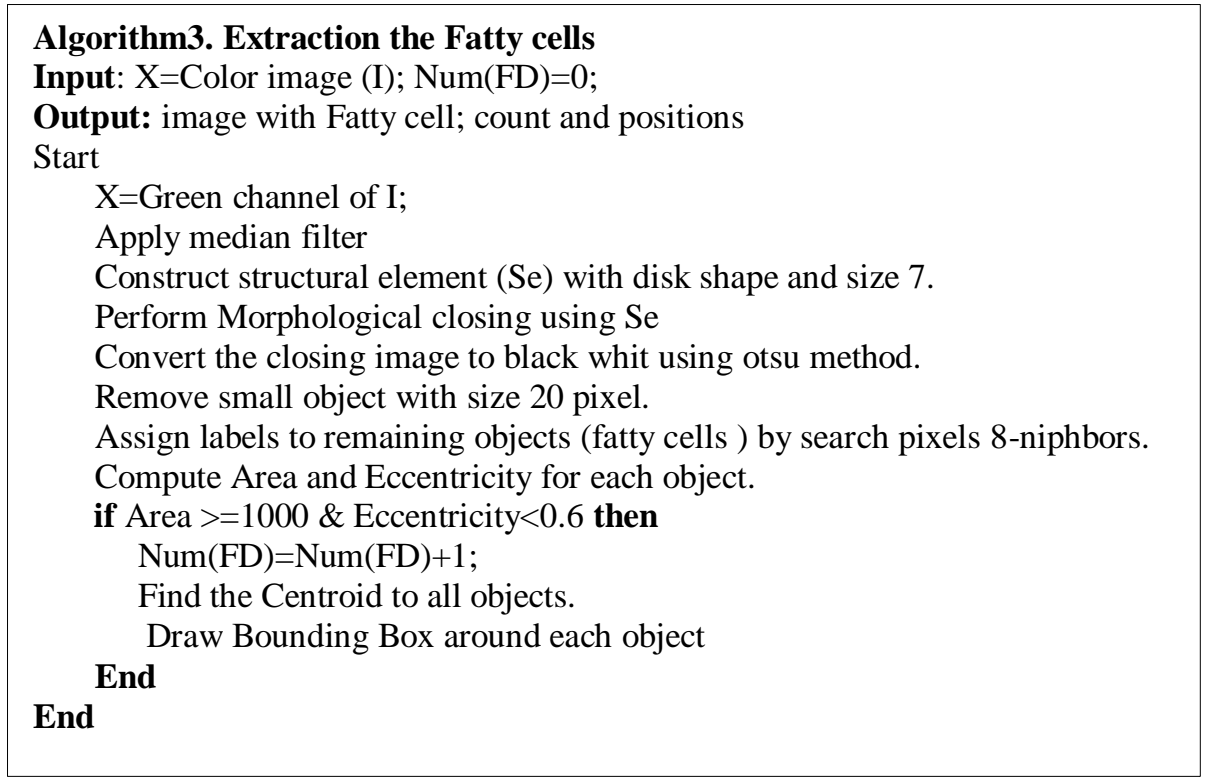

\section{RESULTS AND DISCUSSION}

To verify from the accuracy of our method we made many experimental on dataset of light microscope liver images implies 107 images with 'tif' extension the dataset consist of 7 image is normal case the remaining 100 image have hepatic lesions. Firstly the method test if image normal or not by passing this image in preprocessing then features extraction using GLCM algorithm based on these features the ANN classifier decides whether the image is normal or abnormal. The method success in the determination of the hepatic lesions founded or not with accuracy is $100 \%$.

If abnormal stat founded in the liver tissue image the method repeat treats the color image with three algorithm each one specialize in extract special type of lesions the congestions region detect by apply algorithm 1 result in image with congestions only also the count and positions of these congestion are recorded on it. Figure 4 shows the color image and the extracted blood congestion regions. HHP and HN cells are founded using algorithm 2 by applying it on green channel. Euler number is used to distinguish between the HHP and HN cell Figure 5 shows extracted HHP and HN cell. 

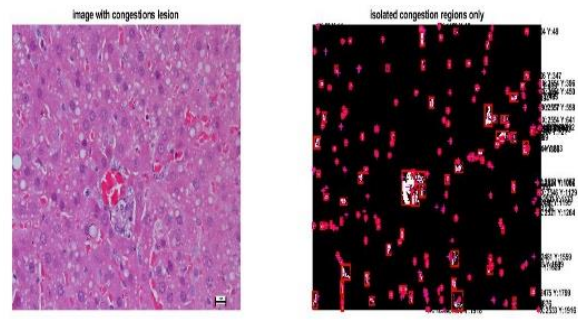

arsim-

Figure 4. Isolated congestion regions
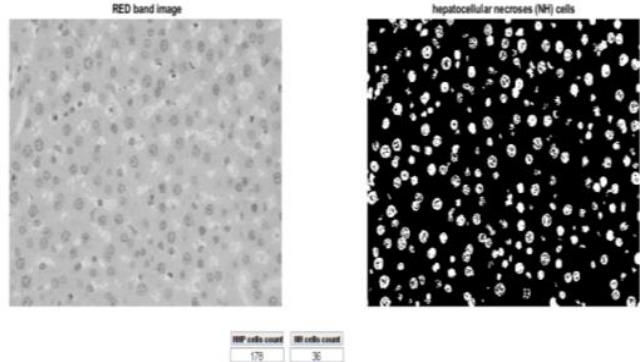

Figure 5. HHP \& HN cells counts

Fatty degeneration cells are isolated from color image by apply morphological closing using disk shape structural element with size 7 then the area and eccentricity of each object are computed to extracted only cells with near circular shape. Figure 6 shows fatty cell on binary image.

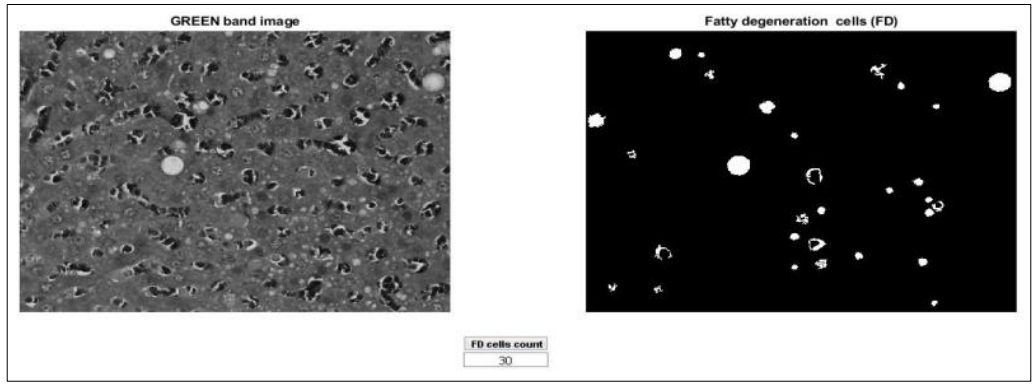

Figur 6.7 fatty cells on binary image

Before compare our method with previous works we must mention all previous work based on CT image our method worked on Light microscope image with four type lesions (congestion, HHP, HN, FD). Table 3 presents comparison between previous methods and the current proposed method, the comparison based on four criteria: type of images, the objective of method and the main techniques used to perform the diagnosing task lastly the accuracy.

Table 3. Comparison Among Methods of Liver Lesions Diagnosing

\begin{tabular}{|c|c|c|c|c|}
\hline Searchers names & Images Type & The objectives & Main techniques & Accuracy \\
\hline S. A. Zaid et al [6] & CT & $\begin{array}{l}\text { Detect Fatty and cancer } \\
\text { detection }\end{array}$ & $\begin{array}{c}\text { Texture analyses and neural } \\
\text { network }\end{array}$ & $96.125 \%$. \\
\hline H. Alahmer and A. Ahmed [10] & CT & $\begin{array}{c}\text { lesion is benign or } \\
\text { malignant }\end{array}$ & $\begin{array}{l}\text { Detect multiple ROIs (inside part, } \\
\text { outside part, and the border part). } \\
\text { SVM used as classifier tool }\end{array}$ & Over $98 \%$. \\
\hline Thayalini Prakash [11] & CT & $\begin{array}{c}\text { Automated liver } \\
\text { segmentation and tumour } \\
\text { detection }\end{array}$ & $\begin{array}{c}\text { Fuzzy C Means } \\
\text { Clustering (FCM) and Gray Level } \\
\text { Co-occurrence Matrix }\end{array}$ & $90 \%$ \\
\hline The proposed method & $\begin{array}{l}\text { Light } \\
\text { microscope } \\
\text { image }\end{array}$ & $\begin{array}{l}\text { Detect normal or ab } \\
\text { normal with } 4 \text { case } \\
\text { Congestion, HN } \\
\text { HHP,FD }\end{array}$ & $\begin{array}{c}\text { GLCM, Texture and } \\
\text { morphological statistical with } \\
\text { ANN }\end{array}$ & $\begin{array}{c}100 \% \\
\text { classification } \\
95 \% \\
\text { diagnosing }\end{array}$ \\
\hline
\end{tabular}

\section{CONCLUSIONS}

Medical image processing is important tool in medicine it can be describe as helper tool to physicians because it benefit in determine the type and amount of disease founded in organs tissue in high accuracy and less time compared with normal diagnosing. This paper introduced new method to diagnose hepatic lesion using Matlab program based on texture and morphological features which are extracted from liver tissue image. 
The method processes two problems firstly it determine whether the image of liver is normal or not based on GLCM features of liver tissue with help of ANN classifier, the second task is determine type of lesion found in cells based on morphological features of cells and the color intensity values for these cells in three band of image Red, Green and Blue. The method achieved accuracy $100 \%$ in classification normality or not and $95 \%$ in determine number and positions of lesions in liver this rates are recorded by compare the lesions found out by current method with lesions detected by pathology expert

\section{REFERENCES}

[1] S. Lüde, "Hepatotoxicity of the phytomedicines Kava kava and Cimicifuga racemosa," University_of_Basel, 2005.

[2] H. Mohammed, "Toxic and histopathological changes of harmful effect of diclofenac sodium on some loose organs in albino rats for twelve weeks," Journal of Medical Science and Clinical Research, vol. 3, no. 3, pp. 4694-4702, 2015.

[3] D. Larrey, "Drug-induced liver diseases," Journal of hepatology, vol. 32, pp. 77-88, 2000.

[4] H. Jaeschke, G. J. Gores, A. I. Cederbaum, J. A. Hinson, D. Pessayre, and J. J. Lemasters, "Mechanisms of hepatotoxicity," Toxicological sciences, vol. 65, no. 2, pp. 166-176, 2002.

[5] R. Teschke, "Drug-induced liver diseases," Zeitschrift fur Gastroenterologie, vol. 40, no. 5, pp. 305-326, 2002.

[6] A. Z. S. A. Zaid, M. W. Fakhr, and A. F. A. Mohamed, "Automatic diagnosis of liver diseases from ultrasound images," International Conference on Computer Engineering and Systems, 2006: IEEE, pp. 313-319.

[7] A. H. Ali and E. M. Hadi, "Diagnosis of Liver Tumor from CT Images using Digital Image Processing," International Journal of Scientific \& Engineering Research, vol. 6, no. 1, 2015.

[8] Y. A. Deore and N. D. Ghuse, "Efficient Image Processing Based Liver Cancer Detection Method," International Journal on Recent and Innovation Trends in Computing and Communication, vol. 4, no. 12, pp. 238-241, 2016.

[9] P. Pruthvi, U. K. Patil, and S. T. Ahmed, "An SVM Approach to Liver Lesion Border Extraction for Liver Cancer Analysis." American Journal Of Computer Science And Information Technolog, vol. 4, no. 1, 2016.

[10] H. Alahmer and A. Ahmed, "Computer-aided Classification of Liver Lesions from CT Images Based on Multiple ROI," Procedia Computer Science, vol. 90, pp. 80-86, 2016, doi: 10.1016/j.procs.2016.07.027.

[11] T. Prakash, "Medical Image Processing Methodology For Liver Tumour Diagnosis," International Journal on Soft Computing (IJSC), vol. 8, no. 3, 2017.

[12] A. Das, U. R. Acharya, S. S. Panda, and S. Sabut, "Deep learning based liver cancer detection using watershed transform and Gaussian mixture model techniques," Cognitive Systems Research, vol. 54, pp. 165-175, 2019.

[13] M. A. Hasan, N. M. Mustapha, A. A. Kadir, and M. Hezmee, "Potential role of Nigella sativa (NS) in abating oxidative stress-induced toxicity in rats: a possible protection mechanism.", IOSR Journal of Pharmacy and Biological Sciences (IOSR-JPBS) Vol. 13, no. 5 , PP 29-42, 2018.

[14] S. Strahl, V. Ehret, H. Dahm, and K. Maier, "Necrotizing hepatitis after taking herbal remedies," Deutsche medizinische Wochenschrift, vol. 123, no. 47, pp. 1410-1414, 1998.

[15] K. M. Saleh, W. K. Zainab, A. Mohammed, and T. A. Alaa, "Toxico-Pathological Study Of Gentamicin By Intramuscular Injection In Experimental Rabbits." Bas.J.Vet.Res.Vol.17, No.2, 2018.

[16] D. Gadkari, "Image quality analysis using GLCM,", Orlando (FL): University of Central Florida 2004.

[17] A. F. H. Alharan, H. K. Fatlawi, and N. S. Ali, "A cluster-based feature selection method for image texture classification," Indonesian Journal of Electrical Engineering and Computer Science, vol. 14, no. 3, 2019, doi: 10.11591/ijeecs.v14.i3.pp1433-1442.

[18] Z. F. Jabr, S. R. Saleh, and A. N. Fasial, "A Hybrid Features for Signature Recognition Using Neural Network," journal of thi-qar science, vol. 6, no. 1, pp. 83-88, 2016.

[19] H. A. Nugroho, I. M. D. Maysanjaya, N. A. Setiawan, E. E. H. Murhandarwati, and W. K. Z. Oktoeberza, "Feature analysis for stage identification of Plasmodium vivax based on digital microscopic image," Indonesian Journal of Electrical Engineering and Computer Science, vol. 13, no. 2, 2019, doi: 10.11591/ijeecs.v13.i2.pp721-728.

[20] S. K. A. Z. F. Jabr, "ECG Heart diseases Diagnosis in Three Cases (Normal, Bradycardia, Tachycardia) by Using GLCM and Fuzzy Logic,", International Journal of Innovative Engineering and Emerging Technology, vol 2, issue 4, 2016.

[21] S. P. Aware, "Image Retrieval Using Co-Occurrence Matrix \& Texton Co-Occurrence Matrix For High Performance," International Journal of Advances in Engineering \& Technology, vol. 5, no. 2, p. 280, 2013.

[22] T. A. Pham, "Optimization of texture feature extraction algorithm," M.Sc.E thesis Delft University of Technology. 2010.

[23] M. A. Shahin, M. B. Jaksa, and H. R. Maier, "Artificial neural network applications in geotechnical engineering," Australian geomechanics, vol. 36, no. 1, pp. 49-62, 2001.

[24] L. Y. Ann, P. Ehkan, M. Mashor, and S. Sharun, "FPGA-based architecture of hybrid multilayered perceptron neural network," Indonesian Journal of Electrical Engineering and Computer Science, vol. 14, no. 2, pp. 949-956, 2019.

[25] Z. F. Jabr, R. H. Hussain and S. R. Saleh, " Arrhythmia Detection Based On Combination Of Freeman Chain Code And First Order Texture Features," Journal of Theoretical and Applied Information Technology, vol. 96, no. 1, 2019. 


\section{BIOGRAPHIES OF AUTHORS}

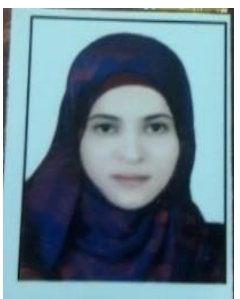

Zamen F. Jabr Lecturer at the college of Education for Girls University of Dhi-Qar holds a master's degree in computer science from the University of Basra College of Science in 2012 in the specialization of image processing and pattern recognition. She holds a bachelor's degree in computer science from the University of Dhi-Qar Faculty of Science in 2005. She has many research in the field of pattern recognition and image processing and is interested in the branch of artificial intelligence and has also researched in this field.

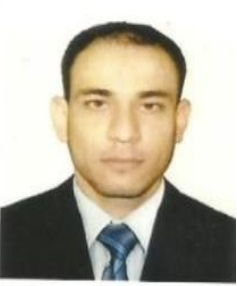

Mohammed A. Hasan employed as a demonstrator by Department of Pthology and Poultry diseases, Faculty of Vetrinary Medicine, Universtiy of Basra for two years. In 2009, I started my pos-graduated study for the degree of Master of Science in Pathology in the Department of Pathology at the Faculty of Vetrinary Medicine, Universtiy of Basra. While in 2014 I started my pos-graduated study for Ph.D. in Molecularpathology/Liver cancer in the Department of Pathology \& Microbiology at Biomedical Scirnce, Universiti Putra Malaysia. In 2018, I obtained My Ph.D. following this; I served as lecturer in Biology Department, College of Education for women/ Universtiy of Thi-Qar. 